\title{
Hans Cornelius: Leben, Denken, Wirkung
}

\section{Hans Cornelius: Life, Thought and Influence}

\section{Daniel Pucciarelli}

Universidade Estadual de Minas Gerais (UEMG)

Orcid 0000-0002-9053-6444

arelli@gmail.com

\section{Eduardo Soares Neves Silva}

Universidade Federal de Minas Gerais (UFMG)

Orcid 0000-0002-8601-7617

esnsilva@gmail.com

Zusammenfassung: Die Arbeit liefert einen Überblick über das Leben, das Denken und die philosophische Wirkung des Philosophen Hans Cornelius. Ausgehend von einer kurzen Rekonstruktion seiner Biographie exponiert der Text die Hauptideen, die er in seinen philosophischen Schriften entwickelt. Den Wahrnehmungs- und Dingbegriffen Cornelius wird in diesem Zusammenhang Vorrang gegeben, da diese Begriffe in der Regel als den originellsten Beitrag Cornelius' angesehen werden. Danach stellt der Aufsatz die Kritik Husserls an Cornelius und die Quelle ihrer philosophischen Differenzen dar. Zuletzt wird noch auf die Wichtigkeit Cornelius' für die Entstehung der sogenannten „Frankfurter Schule“ reflektiert.

Keywords: Münchener Phänomenologie, Psychologismus, Husserl, Frankfurter Schule 
Abstract: The paper provides an overview of the life, the thinking and the philosophical influence of the philosopher Hans Cornelius. After a brief reconstruction of his biography, the text exposes the main ideas he develops in his philosophical writings. Our priority is given to Cornelius' concepts of "perception" and the "thing", as his reformulation of these concepts have generally been regarded as his most original contributions to the history of philosophy and phenomenology. The essay then presents Husserl's criticism of Cornelius and the source of their philosophical differences. Finally, we reflect on the importance of Cornelius for the emergence of the so-called "Frankfurt School".

Keywords: Munich Phenomenology, Psychologism, Husserl, Frankfurt School

\section{Einleitung}

Hans Cornelius teilt das melancholische Schicksal jener Denker, die der kompletten Unbekanntheit nur nicht angehören, weil sie anderen Denkern wichtig waren. Hat man überhaupt von ihm gehört oder gelesen, dann in der Regel durch Husserl oder Adorno und Horkheimer, die sich auf ihn teils mit Bewunderung, teils kritisch, aber immer mit Interesse beziehen. Seine Relevanz wird dementsprechend durch die Relevanz anderer gemessen; wenn überhaupt, wird seine Arbeit höchstens als Bindeglied, kaum für sich selbst gelesen. Der vorliegende Aufsatz möchte diese Diagnose zwar nicht von vornherein revidieren, sondern kann ihre eigene Existenzberechtigung in erster Linie aus diesem Umstand ableiten. Denn zur Relevanz eines philosophischen Denkens gelten nicht nur rein innertheoretischen Elemente, sondern auch äußerliche Bedingungen und Umstände, die durchaus kontingent sind. Sofern Cornelius für jene Denker ersten Ranges wichtig ist, wird auch eine ausführliche Auseinandersetzung mit seinem Denken nicht nur begründet, sondern wohl auch für deren volles Verständnis erfordert. Eine derartige Auseinandersetzung liegt aber noch kaum vor.

Doch auf diesem Weg kann die vorliegende Untersuchung ebenso die Elemente entwickeln, damit jene Diagnose bes- 
ser begründet oder widersprochen werden kann. So unternimmt sie den Versuch, Cornelius möglichst in seinem eigenen Recht zu behandeln, um zu einer autonomen Betrachtung seines Denkens beizutragen. Sie liefert erstens ein Porträt der Person Hans Cornelius (2), um dann zu einem ausführlichen Panorama über sein Denken überzugehen (3). Erst dann versucht die Untersuchung, die Auseinandersetzung mit Husserl zu thematisieren (4). Zum Schluss wird noch auf die Relevanz Cornelius' für die Autoren der sogenannten „Frankfurter Schule“ reflektiert (5).

\section{Hans Cornelius: Ein Porträt}

Hans Johannes Wilhelm Cornelius ist 1863 in München geboren $^{1}$. Studiert hat er Mathematik, Physik und Chemie in München, Berlin und Leipzig. Von seinen Münchener Professoren berichtet er lediglich, dass an der dortigen Universität „sich damals für Mathematik und besonders für Physik in den ersten Stellen völlig minderwertigen Lehrkräften befanden“ (Cornelius, 1921, S. 84). In Berlin studiert er 1882 und 1883 bei Weierstraß und Kirchhoff; Helmholtz aber, der allbekannte Universalgelehrter seiner Zeit, „blieb [Cornelius] gleichgültig; [dieser] konnte [sich] in seinen stets unvorbereiteten, unordentlich vorgetragenen theoretischen Vorlesungen nicht aufnahmefähig erhalten“ (Ebd., S. 85.). Schließlich studiert Cornelius reine Mathematik bei dem großen Mathematiker Felix Klein in Leipzig und zielt darauf ab, dort sein Studium abzuschließen; ,jedoch nach zwei Monaten kam [er] nach Hause, überarbeitet und überzeugt, daß die mathematische Arbeit nicht [s]eine Sache sei“. Zu dieser Zeit hatte Cornelius schon eine anfängliche, experimentierende Beschäftigung auch mit der Philosophie hinter sich - vor allem mit der Kritik der reinen Vernunft und mit dem Denkgebäude Schopenhauers -, sodass er dann seinem Vater erklärte, „daß [er] nur zur Musik oder zur Philosophie als Lebensberuf [sich] entschließen könne“" (Ebd.). Sich aber für die Chemie entscheidend, promovierte Cornelius 1886 als Chemiker mit einer Arbeit über die Synthese des Orzins aus Zitronensäure in München und arbeitet zwei Jahre lang im $1 \quad$ Alle biographischen Informationen über Hans Cornelius stammen dem von Cornelius publizierten autobiographischen Text Cornelius, 1921. 
Laboratorium vom Chemiker Adolf von Baeyer. Seine erste Habilitationsschrift über die Axiome und Hypothesen der exakten Wissenschaften aber, die Cornelius der naturwissenschaftlichen Sektion der Philosophischen Fakultät in München eingereicht hatte, wurde von der Fakultät aufgrund „ungenügender philosophischer Vorbildung“ zurückgewiesen (Ebd., S. 86).

Mit dem Scheitern seines ersten Habilitationsversuches widmet sich Cornelius intensiver der Philosophie zu. Wohl aufgrund seiner eigenen intellektuellen - naturwissenschaftlichen - Laufbahn, aber auch zweifelsohne im Sinne des dann in nachidealistischem Deutschland herrschenden Zeitgeistes orientiert sich Cornelius durchaus an einer Art Neukantianismus, der der dann höchstfortgeschrittenen naturwissenschaftlichen Methode gewachsen sein könne. Er beschäftigt sich zunächst „mit der neueren und neuesten Philosophie, vor allem mit der englischen und deutschen psychologischen Literatur" (Ebd., S. 86-7) und zielt bereits darauf ab, eine „von allen illegitimen Begriffen freie[n] Philosophie“, die auf der ,sichere[n] Grundlage metaphysikfreier Naturerkenntnis" ruhe (Ebd.), zu begründen. Diesem philosophischen Programm, das ja in vielerlei Hinsicht die deutsche - und wohl europäische - Jahrhundertwende tief mitprägt, blieb Hans Cornelius lebenslänglich treu. Philosophisch betreibt er stets, mithilfe der Psychologie, „metaphysikfreie" und systematische prima philosophia. Seine ersten rein philosophischen Schriften - die zweite, nun erfolgreiche Habilitationsschrift Versuch einer Theorie der Existentialurteile (1894) und Psychologie als Erfahrungswissenschaft (1897) -, die die Aufmerksamkeit und Kritik von so unterschiedlichen Lesern wie Edmund Husserl und Wladimir Lenin veranlassen, legen Zeugnis von einem ersten Versuch ab, ein derartiges Programm durchzuführen. Dabei versucht er eben

eine von allen dogmatischen Voraussetzungen freie Wissenschaft von den unmittelbar gegebenen Tatsachen des Bewußtseinsverlaufens, also eine metaphysikfreie Psychologie zu begründen (...), die als Unterbau für alle weitere Philosophie und für alle Erfahrungswissenschaft dienen sollte (Cornelius, 1921, S. 87).

Dass Cornelius dem Geiste des Positivismus gewissermaßen niemals ganz fremd gewesen ist, beweist die Tatsache, dass 
er 1931, am Ende seines intellektuellen Lebens also, einen Aufsatz (Cornelius, 1931) in der klassischen Zeitschrift Erkenntnis, von R. Carnap und H. Reichenbach herausgegeben, veröffentlicht.

Nach seiner Habilitation wirkte Cornelius etwa zehn Jahre lang als Privatdozent, und noch etwa fünf als außerordentlichen Professor für Philosophie in München. Zu dieser Zeit schreibt Husserl eine lange Rezension über Versuch einer Theorie der Existentialurteile (1894) und übt Kritik an Cornelius' Psychologie in seinem ersten, 1906 erschienenen Hauptwerk Logische Untersuchungen. Die Rezension und Auseinandersetzung Husserls veranlassen dann Cornelius, sich mit dem Denken Husserls gründlich zu befassen und anschließend mit ihm 1906 im sehr kurzen, aber bedeutenden Briefwechsel zu stehen. Im Laufe dieser Auseinandersetzung entsteht Cornelius' drittes philosophisches Werk: Einleitung in die Philosophie (1903), die seine philosophische Theorie mit einer Philosophie der Geschichte der Philosophie ausführt. Die Entstehung und Formierung des eigentlich philosophischen Denkens von Hans Cornelius dürfte man also wohl zwischen 1894, da er den Versuch einer Theorie der Existentialurteile der Philosophischen Fakultät der Münchener Universität als Habilitationsschrift einreicht, und 1903, da er sein Werk Einleitung in die Philosophie veröffentlicht. Außer diesen zwei Werken und der bereits erwähnten Psychologie als Erfahrungswissenschaft hat Cornelius erst viel später, 1916, nur noch ein viertes Werk - die Transzendentale Systematik - veröffentlicht, das als rein philosophisch bezeichnet werden dürfte und eben hauptsächlich eine Systematisierung der Gedankengänge der anderen drei Bücher enthält ${ }^{2}$. Zwar haben sich sehr wenige Interpreten mit seinem Denken gründlich befasst, aber die wenigen, die dies getan haben, oszillieren nämlich zwischen der Behauptung, sein Denken sei bereits 1897 mit der Psychologie als Erfahrungswissenschaft vollständig gebildet, und der Auffassung, dass es mindestens noch bis 1903, wenn nicht bis 1916, wesentlichen Veränderungen unterliege. $\mathrm{Zu}$ dieser Frage, wie zur Husserl-Cornelius-Debatte, kommen wir noch ausführlicher zurück.

$2 \quad$ Cornelius selbst hat die Transzendentale Systematik als ein zwar wichtiges, doch als „Ergänzungswerk“ gekennzeichnet: „Wer meine Philosophie kennen lernen will, möge zuerst meine 'Einleitung' lesen; in der 'Transzendentale Systematik' wird er einige wesentliche Ergänzung dazu finden“, Cornelius, 1921, S. 93. 
Auch zur Cornelius' Münchener Zeit nimmt er an der sich um Theodor Lipps bildenden Gruppe von Philosophen und Psychologen teil - dem sogenannten Psychologischen Verein -, die dann später als die „Münchener Phänomenologie“"3 Bekanntschaft erringen würde; zwar wird er kein richtig aktives Mitglied des Vereins sein, aber sah in der Gruppe unter anderen eine Möglichkeit für seine Berufung als Ordinarius für Philosophie, da Theodor Lipps 1909 der Gruppe, und dann 1914 der Fakultät ausgetreten sein würde. Auch deshalb lehnt Cornelius 1908 einen Ruf nach Halle a. S. als Ordinarius ab, aber seine Hoffnungen konkretisieren sich, auch aufgrund von politisch-bürokratischen Umständen und von Streitigkeiten mit Lipps (wohl der bekannteste Anhänger des Psychologismus im damaligen Deutschland), letztinstanzlich nicht ${ }^{4}$. Aus diesem Grunde entschließt er sich, 1910 eine ordentliche Professur für Philosophie an der Akademie für Sozialwissenschaften (späten Johann-Wolfgang-von-Goethe Universität) in Frankfurt am Main zu übernehmen. Seine Antrittsvorlesung behandelte das aufschlussreiche und zu jener Zeit - und wohl immer noch heute - kontroverse Thema: „Die Erkenntnis der Dinge an sich“.

Während seiner Münchener Zeit beschäftigt sich Cornelius und publiziert auch zu Kunstphilosophie - wie sein 1908 erschienenes Buch Elementargesetze der bildenden Kunst: Grundlagen einer praktischen Ästhetik -, es ist aber während seiner Frankfurter Zeit, die solche nicht rein philosophischen Beschäftigungen und Veröffentlichungen intensiver und wohl auch fruchtbarer werden. Neben der kontinuierlichen praktischen Beschäftigung mit Bildhauerei und dann mit Malerei entstehen in seiner Frankfurter Zeit - die von 1910 bis zu seiner Emeritierung 1928

\footnotetext{
3 Über die heute eher unbekannte „Münchener Phänomenologie“, vgl. Smid, 1982.

4 „Schon damals und noch weit mehr in der unmittelbar folgenden Zeit wurden die Verhältnisse in München von Tag zu Tag unerfreulicher (...) durch die immer krankhafter ausartende Mißgunst meines Oberkollegen Theodor Lipps, der in [Cornelius] mit Recht einen wissenschaftlichen, sehr mit Unrecht einen persönlichen Gegner sah“. Cornelius, 1921, S. 91.
} 
andauert - sein politisches Buch Völkerbund und Dauerfrieden ${ }^{5}$ (1919), seine Kunstpädagogik: Leitsätze für die Organisation der künstlerischen Erziehung (1921) wie seine Werke Vom Wert des Lebens (1923) und Die Aufgabe der Erziehung (1928).

Nach seiner Emeritierung im Jahre 1928 veröffentlicht Cornelius keine weiteren originellen Arbeiten. Abgesehen von dem bereits erwähnten, 1931 in der Zeitschrift Erkenntnis publizierten Aufsatz befasst er sich theoretisch mit Zusammenfassungen und neuen Systematisierungen seines philosophischen Denkens, wie seinem Beitrag im interessanten Sammelbande Deutsche Systematische Philosophie nach ihren Gestaltern (1934). Seine Professur an der Frankfurter Universität wurde kurzfristig von Max Scheler, dann vom Theologe und Religionsphilosoph Paul Tillich übernommen. Gestorben ist Hans Johannes Wilhelm Cornelius 1947 in Gräfelfing bei München.

\section{Denken}

So hat Cornelius sein eigenes Denken retrospektiv, 1923, charakterisiert: „Unter den dogmatischen Voraussetzungen, die dem Streben nach letzter Klarheit im Wege stehen,

$5 \quad$ Politisch war Cornelius, wie es in dem in Völkerbund und Dauerfrieden formulierten Programm zu lesen ist, ein ausgesprochener Internationalist und Kriegsgegner (das Buch wurde noch während des Ersten Weltkrieges konzipiert). In dieser Schrift entwickelt er die im Sinne der damaligen Weltpolitik radikale Idee eines Föderalismus aller europäischen Länder als einzige Möglichkeit zur Erreichung einer dauerhaft pazifistischen Lösung für die inneren politischen Konflikte Europas. In seinen eigenen Worten: „Ich konnte in dem Wahnsinn des Weltkrieges nichts anderes sehen, als die Folge der allgemein herrschenden Unklarheit über die fundamentalen Bedingungen des sozialen Daseins. Die klare Erkenntnis dieser Bedingungen seitens der Regierungen mußte die Beendigung des Krieges und den Zusammenschluß der feindlichen Nationen zu föderativen übernationalen Staatsbildungen zur Folge haben, analog wie in der Schweiz und in Amerika die Föderativstaaten aus vorher feindlichen Bestandteilen zusammengeschmiedet worden waren (...) Nicht ein Mitteleuropa im Sinne Naumanns, sondern ein Gesamteuropa oder zum mindesten ein Kontinentaleuropa, nicht als Staatenbund, sondern als Bundesstaat mit einheitlichem Wirtschaftsgebiet, mit einheitlicher Diplomatie und Militärgewalt war das Gebilde, durch welches nicht nur die Gründe für alle 'nationalen' Kriege - in erster Linie die elsaß-lothringische Frage und alle andern wirklichen oder vermeintlichen 'Irredenta'-Probleme ausgeschaltet, sondern auch alle Möglichkeiten für kriegerische Störungen in Europa endgültig beseitigt werden konnten“. Cornelius, 1921, S. 92. 
zeigte sich bereits oben als die folgenschwerste diejenige des Dingbegriffs. Von der Forderung der Klärung dieses Begriffs ist die Entwicklung meiner Philosophie ausgegangen“ (Ebd., S. 15). In der Tat synthetisiert das Zitat den Großteil dessen, was Cornelius im Laufe seines Lebens philosophisch getan hat. Zunächst: Cornelius möchte sein Philosophieren innerhalb einer großen aufklärerischen Tradition - die wohl mindestens auf Descartes und die Anfänge der neuzeitlichen Philosophie zurückgreift - platziert sehen, die die Hauptanliegen der Philosophie in dem Streben nach letzter Klarheit und so in der Gewinnung einer möglichst voraussetzungslosen Lehre sieht. Das Wort „dogmatisch“ wird von Cornelius eben im aufklärerischen Sinne eines Denkgebäudes verwendet, dessen Voraussetzungen und Grundlagen nicht stringent geprüft worden waren und somit nicht klar und distinkt nachvollziehbar sind. Darin, wie in vielen anderen Aspekte seines Denkens wie etwa in der Rede von „metaphysischen Scheinproblemen“ und dem allgemeinen Anspruch auf Wissenschaftlichkeit für die Philosophie, ist Cornelius, wie bereits erwähnt, dem philosophischen Geist seiner Zeit gar nicht fremd. Mit den sogenannten „metaphysikfreien“ und „wissenschaftlichen Philosophien“ - von dem Frühen Positivismus bis zum Wiener Kreis - war Cornelius in mehr oder minder fruchtbarer Beziehung. An das Denken von Mach und Avenarius etwa, besonders das sogenannte „Prinzip der Denkökonomie“ (Mach) bzw. das „Prinzip des kleinsten Kraftmaßes" (Avenarius) betreffend, hat er mindestens bis 1903 ausdrücklich angeknüpft ${ }^{6}$.

$6 \quad$ Für Rollinger (1991) ist das Denken Cornelius' bereits in der Psychologie als Erfahrungswissenschaft wesentlich gebildet; danach variiert ihm zufolge bloss jeweils die verwendete Terminologie. Jay (1996) und Petazzi (1979) argumentieren dagegen, dass Cornelius zu seiner eigentlichen Denkweise erst infolge der Auseinandersetzung mit Husserl und der Loslösung von Mach und Avenarius tatsächlich gelangt. Aus dem Standpunkt der Wirkungsgeschichte seines Denkens hat Rollinger wahrscheinlich recht, da Cornelius interessanterweise von Husserl und Lenin immer als Anhänger von Mach und Avenarius gesehen wurde. Pettazzi schreibt diesbezüglich: „I libri di Cornelius sono oggi dimenticati e un velo polveroso li ricopre nelle biblioteche. A suo tempo essi dovettero però godere di una certa notorietà, tanto che vengono citati in opere come Materialismo et empiriocriticismo di Lenin e Richerche logiche di Husserl. In ambedue i casi Cornelius viene considerato un sostenitore e continuatore delle posizioni di Mach e Avenarius, e su questa base aspramente criticato“. Pettazzi, 1979, S. 40. 
Eine voraussetzungslose und metaphysikfreie - oder, wie Cornelius sich auch ausdrückt: von naturalistischen Voraussetzungen befreite - Philosophie kann sich dann nach Cornelius lediglich auf dasjenige Material stützen und aufgebaut werden, das sich jederzeit dem Bewusstsein mit unmittelbarer Klarheit und Evidenz darstellt: das, was Cornelius meistens das unmittelbar Gegebene nennt. Diesbezüglich ist er in vollem Einverständnis etwa mit der Tradition des radikalen Empirismus von David Hume und William James - in den Logischen Untersuchungen beispielsweise hat Husserl Cornelius als den radikalsten „modernen Humeaner“ gekennzeichnet (Husserl, 1921, S. 211ff.). In wesentlichem Unterschied mit der Tradition des Empirismus - und auch wohl der Transzendentalphilosophie - aber dürfte diejenige Lehre Cornelius' gezählt werden, derzufolge die empirische Erfahrung keine minderwertige, sondern eine allgemeingültige Erkenntnisquelle darstellt. Dies präsentiert der Philosoph namentlich als den originellsten Hauptpunkt seiner Lehre: „Ein Hauptpunkt meiner Lehre, durch den sie sich zu allem Herkommen in Gegensatz stellt, ist der Nachweis, daß Erfahrung durchaus keine minderwertige Erkenntnisquelle ist, vielmehr uns allgemeingültige Erkenntnis zuführt" ${ }^{\text {"7 }}$. In der Tat ist diese Lehre, soweit sie wirklich begründet werden kann, sehr originell. Dies macht wohl die allererste Besonderheit der Philosophie Cornelius' aus, denn mit dieser Lehre kann er nichts weniger als den Anspruch erheben, eine radikal-empiristisch, also lediglich auf klaren und evidenten Bewusstseinsinhalten bzw. Gegebenheiten gestützte Philosophie als System aufzubauen, die dann nicht in Skepsis einmünden muss. Dieses Programm einer systematischen Neubegründung der Philosophie mithilfe der in diesem Sinne verstandenen Psychologie hat Cornelius, wie gesagt, wohl immer durchzuführen versucht; sein Werk Psychologie als Erfahrungswissenschaft, das dieses Anliegen zum ersten Male systematisch und ausführlicher dars-

\footnotetext{
$7 \quad$ Cornelius, 1921, S. 14: So referiert Husserl diese Lehre Cornelius‘: „Wir besitzen in der äußeren wie in der inneren Wahrnehmung durchaus gleichgeordnete und keiner Täuschung ausgesetzte Grundlagen und Quellen der Erkenntnis; ja mit Rücksicht auf die Schwierigkeiten der inneren Beobachtung ist die äußere Wahrnehmung geradezu als die sicherste Erkenntnisquelle zu betrachten“. Husserl, 1979, S. 358.
} 
tellt, möchte er als philosophische Grundwissenschaft oder gar als reine Phänomenologie verstanden wissen ${ }^{8}$.

Obwohl erst systematisch und ausführlich in der Psychologie als Erfahrungswissenschaft (1897) dargestellt, werden einige der oben referierten Kernelemente des Denkens Cornelius bereits in seiner Habilitationsschrift Versuch einer Theorie der Existentialurteile ansatzweise dargestellt. In diesem Werk befasst sich der Autor nicht nur mit einer Theorie der Existentialurteile, sondern, wie Husserl bemerkt, mit einer Urteilstheorie im Allgemeinen, die aber erst am Ende der Schrift vollständig entwickelt wird. Dabei setzt sich Cornelius hauptsächlich mit der Tradition des radikalen Empirismus wie mit der Brentanoschule auseinander. Das allgemeine Ziel der Schrift besteht darin, die Urteilstheorie ausgehend lediglich vom unmittelbar Gegebenen bzw. vom „elementaren Wahrnehmungsurteil" neu zu begründen, sodass alle anderen Urteilsklassen vom Wahrnehmungsurteil unmittelbarer - klarer und distinkter - Gegebenheiten abgeleitet werden. Dazu muss Cornelius dementsprechend den eigentlichen Begriff der inneren und äußeren Wahrnehmung derart konzipieren, dass eine jegliche Transzendenz zum Bewusstsein dezidiert ausgeschlossen bleibt. Wahrnehmung, gleichgültig nun ob innere oder äußere, ist nach Cornelius (und hier noch im Sinne der Brentanoschule) stets auf einen rein immanenten Bewusstseinsinhalt bezogen, der sich im Bewusstseinsverlauf befindet. Das Wahrnehmen wird somit zu einer Art Innewerden eines bereits vorhandenen und rein immanenten Bewusstseinsinhalts:

Erklären wir in Übereinstimmung mit einer ziemlich verbreiteten Terminologie die innere Wahrnehmung allgemein als die Wahrnehmung psychischer Phänomene, so erscheint es nur konsequent, die äussere Wahrnehmung als entsprechende Wahrnehmung physischer Phänomene zu definieren. Wenn nach dieser Definition die innere und die äussere Wahrnehmung sich nur durch die wahrgenommenen Inhalte unterscheiden, so wird über die Bedeutung des Wortes Wahrnehmung auch bei der äussern Wahrnehmung kein

$8 \quad$ „Ich sehe heute ein, daß der Titel des Buchs für die Fachgenossen mehr eine Verdunkelung als eine Andeutung seiner Absicht sein mußte. Ich hätte es - mit entsprechender Umformung seiner Terminologie - lieber als 'reine Phänomenologie' oder als 'philosophische Grundwissenschaft' bezeichnet sollen". Schmidt, 1921, S. 85. 
Zweifel bestehen können: auch hier ist das Wahrnehmen nichts anderes als das Vorfinden, Innewerden oder Bemerken eines gegenwärtig als Inhalt unseres Bewusstseins vorhandenen physischen Phänomens (Cornelius, 1894, S. 8).

Dass der Autor somit die Begründung einer reinen Immanenzphilosophie im Sinne hat, lässt er keine Zweifel bestehen, indem er den Begriff physischer Phänomene derart erläutert:

Insbesondere darf mit dem Ausdrucke 'physischer Phänomen' nicht der Gedanke an 'ausser-psychische' Existenzen, an die Objekte im Sinne der Physik verbunden werden: das physische Phänomen bezeichnet stets nur einen Empfindungs- oder entsprechenden Vorstellungsinhalt als solchen, nicht aber irgend eine äussere Ursache, einen Reiz, welcher diesen Inhalt hervorbringt (Cornelius, 1894, S. 8.).

Dies bedeutet dann letztinstanzlich, dass auch die genannten physischen Phänomene „wesentlich psychische Inhalte [sind], da sie, als Phänomene, nur innerhalb eines wahrnehmenden Bewusstseins denkbar sind“" (Ebd.). Ein Wahrnehmungsbegriff dagegen, der den Verweis auf Bewusstseinstranszendentes oder gar ,irgend welchen bewussten oder unbewussten Schluss auf eine Ursache dieses Phänomens, einen Reiz, eine Substanz oder ein Ding an sich“ zuließe (Ebd., S. 9), wird von Cornelius als die „weitverbreitet[e] metaphysisch[e] Auffassung des Begriffs der äusseren Wahrnehmung" charakterisiert (Ebd.).

Diesem radikal immanenten Wahrnehmungsbegriff gemäß, auf dem Cornelius seine Urteilstheorie basieren will, wird etwas dann wahrgenommen, wenn ein bereits vorhandener Bewusstseinsinhalt als solchen bemerkt wird; bemerkt wird er dann, wenn er vom Gesamtinhalt, der das Bewusstsein konstituiert, unterschieden wird - oder, besser gesagt, der das Bewusstseins letztinstanzlich ist ${ }^{9}$. Wahrnehmen wird zum Bemerken eines Bewusstseinsinhaltes; Bemerken wird seinerseits zu nichts anderem als dem Unterscheiden dieses gegenwärti-

$9 \quad$ Aus dieser Neubestimmung des Wahrnehmungsbegriffs kann man bereits in aller Klarheit einsehen, dass Cornelius einer der strengsten Gegner der sogenannten Atomistik bzw. atomistischen Psychologie gewesen ist. Rudolf Eisler beispielsweise behandelt Cornelius so: „Atomistische Psychologie wird jede psychologische Richtung genannt, welche voraussetzt, daß das Psychische aus ursprünglich bestehenden, isolierten Elementen aufbaut. Dagegen besonders H. Cornelius“. Eisler, 1904, Antrag: „Atomistische Psychologie“. 
gen Inhaltes von vorher wahrgenommenem Gesamtinhalt des Bewusstseinsverlaufs. In diesem Sinne ist dieses Wahrnehmen bzw. dieses Bemerken für Cornelius vom Urteil nicht zu unterscheiden: „Das (elementare) Wahrnehmungsurteil“, schreibt er am Ende des ersten Kapitels dieser Schrift,

besteht somit wesentlich in der Erkenntnis des gegenwärtigen Inhaltes als eines vom vorhergehenden Inhalt verschiedenen. Diese Erkenntnis aber ist von der Thatsache des Vorfindens des betreffenden Inhaltes, seinem Erscheinen oder Vorgestelltwerden in der üblichen weiteren Bedeutung des letzteren Wortes nicht unterschieden; wir konnten daher einer Trennung des Urteilsaktes im elementaren Wahrnehmungsurteil vom vorstellenden Akte nicht zustimmen (Cornelius, 1894, S. 31).

Das so konzipierte elementare Wahrnehmungsurteil bildet wohl die Basis der Urteilstheorie, die Cornelius in dieser Schrift entwickelt. Die anderen Urteilsklassen, die auf dem Wahrnehmungsurteil basieren oder ihn voraussetzen, subsumiert Cornelius unter die Kategorie der symbolischen Urteile. Während die Wahrnehmungsurteile je auf gegenwärtige Objekte gerichtet sind, betreffen die symbolischen Urteile (Erwartungsurteile und Gedächtnisurteile) nicht gegenwärtige Objekte, die ihrerseits im Bewusstseinsverlauf durch ein Symbol (wie beispielsweise ein Sprachsymbol) stets vertreten sein müssen und somit auf irgend Wahrgenommenes hinweisen. Dies erlaubt bestimmte Vergleichungsrelationen - Relationen von Ähnlichkeit oder Unähnlichkeit - und mit ihnen bestimmte Einordnungskriterien:

Damit wir über ein nicht gegenwärtiges Objekt urteilen können, muss dasselbe irgendwie durch einen gegenwärtigen Inhalt vertreten, repräsentiert sein. Wir wollen diesen gegenwärtigen Inhalt, der an Stelle des nicht gegenwärtigen zu beurteilenden Objektes steht, als Symbol des letzteren bezeichnen: alle Existentialurteile, welche nicht Wahrnehmungsurteile sind, müssen sich hiernach eines Symboles als Repräsentanten ihres logischen Subjektes bedienen und mögen daher im Folgenden kurz als symbolische Existentialurteile bezeichnet sein. Handelt es sich um ein sinnlich wahrnehmbares Objekt, also um einen nicht gegenwärtigen Sinnesinhalt, so dient als Repräsentant desselben in letzter Instanz stets ein diesem Inhalte entsprechendes mehr oder minder bestimmtes Phantasma. Das Prinzip, vermöge dessen dies Phantasma als Symbol jenes Inhaltes erscheint, ist die zwischen beiden bestehende spezifische Vergleichungsrelation, welche, wie jede Vergleichungsrelation, die indirekte Bestimmte ihres eines Fundamentes durch das andere gestattet (Ebd., S. 53). 
Diese so konzipierten Urteilsklassen bilden, beinahe ohne Modifikationen, wohl eine der wichtigsten epistemischen Grundlagen des Gesamtdenkens Cornelius' bis zu seiner Spätphase. Zwar erfahren diese Begriffe terminologische und architektonische Differenzierungen - wie etwa bereits im Werke Einleitung in die Philosophie (1903), in dem sie als die „zwei Kategorien der begrifflichen Bestimmung der Erlebnisse“ auftreten -, ihre logische Funktion bleibt aber als solche größtenteils bestehen. Auch von dieser Urteilstheorie ausgehend meint Cornelius dann, die mit naturalistischen Voraussetzungen kontaminierten Begriffe der Philosophie wie beispielsweise und exemplarisch den Dingbegriff zu reinigen.

Bereits in der Psychologie als Erfahrungswissenschaft (1897) - im klaren Anschluss an den Versuch einer Theorie der Existentialurteile (1894) - spricht Cornelius von einer notwendig zu leistenden „Umbildung des Dingsbegriffs“ (Cornelius, 1897, S. 243) und definiert das Ding somit als den „gesetzmäßigen Zusammenhang seiner Erscheinungen" - eine Bezeichnung, die er seitdem immer wieder verwendet wird ${ }^{10}$. Mit der Lehre des Dings als „gesetzmäßigen Zusammenhanges seiner Erscheinungen" werden für Cornelius die Probleme der subjektiv-idealistischen und kantisch-transzendentalphilosophischen Dingtheorien zugleich behoben, indem die Unabhängigkeit und Beständigkeit des Dinges (bzw. der Dingwelt) vermittelst lediglich bewusstseinsimmanenter Inhalte, ohne Rekurs also auf „metaphysische“ oder „naturalistische“ Begriffe, gesichert werden. Mit der empirischen Erfahrung werden uns laut Cornelius verschiedenartige Schattierungen des wahrgenommenen Dings geliefert, die zugleich den Regel, das Gesetz des Zusammenhanges seiner Erscheinungen in uns bilden. Das Ding ist also nicht einfach die Summe der Erscheinungen, die wir aktuell

$10 \quad$ Alles, was sich ändern kann, ist wie gesagt die Terminologie. Cornelius verwendet beispielsweise in der Transzendentalen Systematik den Begriff „idealgesetzlicher Zusammenhänge“; „idealgesetzlichen Ergebnisses“ usw. Interessanterweise knüpft Adorno an diese Terminologie an bezüglich des Dingbegriffs und auch in seiner Kritik am Husserls Dingbegriff. Vgl. diesbezüglich unten über seine Promotionsschrift Zur Transzendenz des Dinglichen und Noematischen in Husserls Phänomenologie. 
wahrnehmen (wie etwa der subjektive Idealismus behaupten kann), sondern dieser gesetzmäßige Zusammenhang:

Wir fassen nach diesen [bereits über den Dingbegriff durchgeführten - D.P. u. E.S.N.S.] Auseinandersetzungen in der Behauptung der objektiven Existenz eines Dinges eine Reihe gemachter Erfahrungen und auf diese Erfahrungen gegründeter Erwartungen zusammen: wenn ich sage, dieses oder jenes gegenwärtig nicht wahrgenommene Ding existiere, so ist damit derjenige Erfahrungszusammenhang bezeichnet, den ich kennen lernte, als ich unter bestimmten Bedingungen wiederholt bestimmte Wahrnehmungen machte, - die eben als Wahrnehmungen des betreffenden Dinges bezeichnet wurden - und welcher Zusammenhang mich erwarten lässt, dass ich unter Erfüllung der entsprechenden Bedingungen die eine oder die andere Wahrnehmung dieses Dinges abermals erhalten werde. Die eine oder die andere Wahrnehmung des Dinges: denn wie wir gleichfalls gesehen haben, ist mit dem Dingbegriff selbst ebenfalls der gegenseitige Zusammenhang gewisser, unter bestimmten Bedingungen zu machender Erfahrungen bezeichnet, von welchen jede einzelne als 'Wahrnehmung des Dinges' gilt, insofern wir sie nicht als einzelne Thatsache, sondern eben mit Rücksicht auf jenen Zusammenhang betrachten. ${ }^{11}$

\section{Husserls Kritik}

Über die Urteilstheorie Cornelius' hat Husserl am Ende 1896 zunächst eine lange Rezension geschrieben, die ein paar Monate später vom Autor zusammengefasst worden und 1897 im Rahmen eines „Berichts über deutsche Schriften zur Logik aus dem Jahre 1894" im Archiv für systematische Philosophie

$11 \quad$ Cornelius, 1897, S. 241-2. Um nachzuweisen, dass Cornelius diesem so umgebildeten Dingbegriff lebenslänglich treu geblieben ist, sei nur ein Zitat aus einem 1934 publizierten Text angeführt: „Wie hier am Beispiel des Buches, so läßt sich an jedem beliebigen anderen sinnenfälligen Dinge zeigen, daß in ihm stets ein bestimmter Zusammenhang von Erscheinungen vorliegt, die unter völlig bestimmten Bedingungen von uns wahrzunehmen sind und die wir, sobald wir diesen Zusammenhang erkannt haben, als die Erscheinungen dieses bestimmten Dinges bezeichnen. Wir gebrauchen stets das Wort 'dieses Ding' als gleichbedeutend mit dem, was wir auf Grund dieser Erfahrung und Überlegung als diesen gesetzmäßigen Zusammenhang zu bezeichnen haben. Das Ding ist also nicht anderes als ein solcher Zusammenhang der Erscheinungen ('seiner' Erscheinungen!) nach einem bestimmten Gesetz - dem Gesetz nämlich, nach welchem wir je nach der Erfüllung bestimmter Bedingungen die eine oder die andere der Erscheinungen vorfinden, die wir gemäß diesem ihrem Zusammenhang als die Erscheinung dieses Dinges kennen und benennen." Schmidt, 1921, S. 27. 
erschienen ist. In einem Brief an Paul Natorp erläutert Husserl, dass die zuerst verfasste Rezension derart lang war, weil Cornelius typische Fehler begehe (Husserl, 1979, S. 465). Später, in den Logischen Untersuchungen (1906), hat er die Kritikpunkte dieser Rezension teilweise wieder aufgenommen und Kritik an der Psychologie als Erfahrungswissenschaft von Cornelius geübt. Das ist deshalb als den Grundstein der Husserlschen Kritik und Auseinandersetzung mit Cornelius zu bezeichnen.

So fasst Husserl den oben referierten Versuch einer Theorie der Existentialurteile Cornelius' in der kleinen Rezension zusammen:

Es ist eben, wenn ich recht verstehe, der wesentliche Gedanke der Schrift darin zu suchen, daß Vorstellen und Urteilen, Verneinen ebenso wohl wie Bejahen - bloßes Bemerken sei, schlichtes Vorfinden eines Inhalts, während alle Unterschiede, die wir durch Wahl jener mannigfaltigen Termini andeuten, in den vorgefundenen Inhalte liegen sollen (Ebd., S. 139).

Oder mit anderen Worten, diesmal in der großen Rezension:

Das Hauptfundament der vorliegenden Theorie ist die Identifikation von Bemerken und Unterscheiden; damit soll nicht etwa eine terminologische Festsetzung getroffen, sondern eine wichtige Erkenntnis gewonnen sein: Bemerken eines Inhalts ist Unterschieden desselben vom vorhergegangenen Gesamtinhalt (Ebd., S. 372).

Nun vertritt Husserl in seiner Kritik an Cornelius eine Position, die interessanterweise den rein immanenzphilosophischen Standpunkt Cornelius' als solche in Frage stellt und kritisiert. Mit anderen Worten und zugespitzt formuliert: im Vergleich zu Cornelius' rein monistisch-immanenzphilosophischem Standpunkt vertritt Husserl eine Position, die Raum sogar für mögliche dualistisch angelegte Interpretationen offen lässt. Diese Position ließe sich bezüglich zweier Hauptelemente zusammenfassen:

1) Gegen Cornelius' erkenntnistheoretische Identifikation von Bemerken und Unterscheiden - bzw. von „logischen“ und „anschaulichen" Elementen innerhalb der Urteilslehre - besteht Husserl auf deren eigentlichen und nicht zu umgehenden Du- 
alität. Wenn ein Inhalt bemerkt wird, wird er tatsächlich vom Gesamtinhalt des Bewusstseinsverlaufs unterschieden; dieses Unterscheiden, das mit dem Bemerken eines Inhalts vollzogen wird, setzt aber voraus, so Husserl, dass der Inhalt als solcher bereits im Bewusstseinsverlauf unterschieden bestehe, sonst wäre der Inhalt erst vom Bemerken „konstituiert“, was Husserl nicht zu akzeptieren scheint:

Wo immer ein Inhalt für sich bemerkt wird, da ist natürlich das Urteil richtig, daß er von dem 'vorher wahrgenommenen Gesamtinhalt' verschieden ist; aber daraus folgt nicht, daß das Bemerken in diesem beziehenden Urteil (welches erst die nachträgliche Reflexion herbeiführt) bestehe. Sieht man in dem 'Abheben' eines Inhalts von einem Hintergrund ein eigenartiges Verhältnis, das sogar Abstufungen zuläßt, so habe ich nichts dagegen; aber das Bemerken des Inhalts ist nicht das Bemerken dieses Verhältnisses, sondern der Bestand des letzteren (d. h. nicht der Vollzug der beziehenden oder bemerkenden Tätigkeit, durch die wir es gelegentlich erfassen) ist nur die durch psychologische Reflexion und Induktion erkennbare Vorbedingung für die Bemerkbarkeit jenes Inhalts (Husserl, 1979, S. 140).

2) Gegen den ontologischen Monismus, zu dem die Cornelius'sche Position letztinstanzlich führt, verwendet Husserl auch hier ein eher dualistisch angelegtes Argumentationsmuster, das auf die Dualität von Inhalt und Gegenstand und von „Vorstellen im Sinn des Bemerkens und dem durchaus heterogenen Vorstellen im Sinn des Repräsentierens" hinweist (die Cornelius, so Husserl, mit einander vermengt habe). Hier sieht man bereits die Entstehung der Theorie der Intentionalität, die die einige Jahre später veröffentlichten Logischen Untersuchungen vertreten wird:

Einen Gegenstand sinnlich wahrnehmen, das ist dem Verf. < gleichbedeutend mit: $>$ einen sinnlichen Inhalt bemerken. Ist aber der Baum, den ich jetzt mit wanderndem Blick auffasse, identisch mit den mannigfaltigen und verschiedenen Inhalten, die ich bemerke? (Ebd., S. 374).

\section{Und dann ausführlicher:}

Ich muß mich hier < mit > diesen Andeutungen begnügen und kann nur wiederholt meine Überzeugung aussprechen, daß man sich ernstlicher wird mit dem Gedanken vertraut machen müssen: die Repräsentation sei eine gegenüber dem bloßen Bemerken grundverschiedene 'Weise des Bewußtseins von einem Inhalt', und zwar 
eben die, welche den Inhalt zum Repräsentanten eines Gegenstandes stempelt, der selbst kein Teil, kein Stück und auch kein unselbständiges Moment des 'vorstellenden' Aktes ist, sondern ihm nur 'intentional' einwohnt, d. h. nur auf eine Funktion desselben hinweist, die sich entfaltet in gewissen möglichen Urteilszusammenhängen (Ebd., S. 376).

In den Logischen Untersuchungen hat sich Husserl, wie gesagt, erneut mit Cornelius beschäftigt. In diesem Werk aber konzentrieren sich Husserls Kritikpunkte vor allem auf Elemente des Denkens Cornelius, die bereits in Versuch einer Theorie der Existentialurteile zu finden waren und in der Psychologie als Erfahrungswissenschaft systematischer ausgeführt werden. Es handelt sich hauptsächlich, wie im Falle der oben ausgeführten Überlegungen, um eine Kritik an der „Vermengung von dem, was zum intentionalen Inhalt der Erkenntnis gehört (...), mit dem, was zum intentionalen Gegenstande der Erkenntnis gehört, und dieser beiden wiederum mit dem, was näher oder ferner zur bloßen psychologischen Konstitution des Erkenntniserlebnisses gehört" (Husserl, 1921, S. 212).

Diese Vermengungen, so Husserl, treten bei Cornelius klarer und deutlicher als bei jeden anderen Autoren zutage. Dem Geiste nach folgt die Kritik Husserls das gleiche Argumentationsmuster wie im Falle der Kritik am Werke Versuch einer Theorie der Existentialurteile, wobei Husserl gegen die Abstraktionstheorie von Cornelius argumentiert: stets zielt er darauf $\mathrm{ab}$, Inhalt und Gegenstand, bedeutungsverleihenden Akt und Bemerken voneinander getrennt zu halten und so die „psychologistische Erkenntnistheorie“ Cornelius', die sie mit einander größtenteils identifiziert hatte, zu widerlegen.

Aus diesem Standpunkt lässt es sich erklären, warum Husserl mit der Ansicht Cornelius' nicht einverstanden sein könnte, dass sowohl der Psychologie als Erfahrungswissenschaft als auch den Logischen Untersuchungen grundsätzlich dasselbe Projekt zugrunde liegt. Denn während Husserl eine reine Logik begründen will, versucht Cornelius dagegen eine Begründung der Logik durchzuführen, die eben „nah verwandt mit dem Psychologismus“ $\mathrm{zu}$ betrachten ist, wie Husserl behauptet. Husserls eher dualistisch angelegtes Argumentationsmuster läuft dementsprechend 
darauf hinaus, die eigentliche Grundlage des Psychologismus zu widerlegen, wie diese sich prototypisch bei Cornelius dargestellt hatte. Wie könnte dann Cornelius stets darauf bestehen, dass er und Husserl im Grunde dasselbe Projekt im Sinn hatten? Laut Rollinger einigen die Autoren zwar wichtige programmatische Elemente wie der allgemeine Anspruch auf voraussetzungslose Wissenschaftlichkeit, die aber eher sekundär in Ansehung der konkreten Durchführung ihrer Denkweisen bleiben:

From Cornelius' standpoint, he and Husserl both advocate a fundamental philosophical discipline which is to be 1) presuppositionless, 2) not causal-explanatory, and 3) prior to any distinction between mental and physical. And even though Cornelius is committed to a thorough-going empiricism, he diverges from the empiricist tradition by claiming that empirically derived knowledge can be universally valid. Insofar as his fundamental philosophical science aims at such knowledge about experiences (to be reached by imagining examples) and is not to consist of mere probabilities, this aim is a fourth point on which Cornelius and Husserl agree (Rollinger, 1991, S. 52f.).

\section{Hans Cornelius und die Geburt der Frankfurter Schule}

Cornelius' Wichtigkeit für die Philosophiegeschichte beschränkt sich aber nicht nur auf seine Auseinandersetzungen mit Husserl. Er ist wie gesagt auch durchaus bestimmend für die Entstehung einer anderen, in mancher Hinsicht der Phänomenologie entgegengesetzten Denkrichtung des 20. Jahrhunderts gewesen: die Frankfurter „Kritische Theorie“. Die jungen Max Horkheimer und Theodor Wiesengrund-Adorno verfassten ihre Promotion und Habilitation unter seiner Betreuung (Adorno aber zog die unter Cornelius geschriebene Habilitationsschrift dann letztendlich zurück). Über Cornelius konnte Horkheimer noch 1969, in einem sehr melancholischen, kurz nach dem Tode Adornos in Venedig frei gehaltenen Vortrag namens Kritische Theorie gestern und heute, sagen:

Wir [Horkheimer und Adorno - D. P.] haben den Ersten Weltkrieg erlebt und haben nachher nicht studiert, um Karriere zu machen, sondern weil wir von der Welt etwas kennenlernen wollten. Daß uns dies gelungen ist, und daß wir dann doch die akademische Karriere eingeschlagen haben, hängt damit zusammen, daß wir einen wunderbaren philosophischen Lehrer hatten, nämlich Hans Cornelius, 
den Urenkel des Malers Peter Cornelius, des Freundes von Goethe. Er war Professor, hat aber schon die Kritik an der Universität und an seinen Kollegen geübt, die heute von den Studenten erhoben wird. Ja, er war Professor der Philosophie und hat uns gesagt, um Philosoph zu sein - und das alles steht in der Kritischen Theorie - ist es notwendig, die Naturwissenschaften zu kennen, ist es notwendig, etwas von Kunst zu wissen, von Musik und Komposition. Er selbst hat mir Kompositionsunterricht gegeben. Und nur auf diese Weise, durch seine Hilfe, haben wir einen anderen Begriff von Philosophie, als er heute üblich ist, nämlich, daß sie kein Fach sei, keine Disziplin wie andere Disziplinen (Horkheimer, 1979, S. 336).

Und Friedrich Pollock, ein lebenslanger Freund von Adorno und Horkheimer, hat es etwa zur gleichen Zeit bestätigt, dass Cornelius' „influence on Horkheimer can hardly be overestimated" (Brief vom 24. März 1970 von F. Pollock an Martin Jay, in Jay, 1996, S. 44). Ebenso als Professor könnte Cornelius als eine für seine Schüler prägende Figur gekennzeichnet werden: denn er war, so Pollock weiter, ,a passionate teacher (...), in many ways the opposite of the current image of a German university professor, and in strong opposition to most of his colleagues" (Ebd., in: Jay, 1996, S. 45).

Darüber hinaus war Cornelius, wie kurz erwähnt, ein leidenschaftlicher und vorbildlicher Kenner italienischer Kunst der Renaissance. Wenn tatsächlich seiner philosophischen Ästhetik ein bestimmter Klassizismus oder gar ästhetischer Konservatismus inhaltlich sehr nah geblieben ist - darin im unzweideutigen Gegensatz etwa zu Adorno, der zu seiner Studienzeit in Frankfurt bereits ein höchst passionierter Anhänger der Neuen Musik und als produktiver und frühreifer Musikkritiker tätig war -, so ist trotzdem die große theoretische und philosophische Wichtigkeit, die Cornelius der Kunst im Allgemeinen zuschrieb, von seinen Schülern sicherlich als sehr prägend empfunden worden. „Aber man darf die Tatsache nicht überschätzen“, schreibt Carlo Pettazzi,

daß Cornelius ebenso wie Adorno großes Interesse an der Ästhetik hatte, um nicht zu oberflächlichen Parallelen bei beiden zu gelangen: seinen ästhetischen Ansichten nach war Cornelius Klassizist, ein treuer Gefolgsmann Hildebrands; an der Musik und den bildenden Künsten interessierte ihn eigentlich nur, die Werke scharfsinnig auf 'wissenschaftliche Gesetze' zurückzuführen: entsprechend der Erfüllung dieser Gesetze bestimmte sich für ihn ihr ästhetischer Wert (Pettazzi, 1977, S. 30). 
Über die eigentlich philosophische Tragweite dieses Einflusses kann man sagen, dass sich seine Schüler - und hier vor allem der junge Adorno - zunächst als Fortführer seines Projekts sahen. Denn es ist eben die philosophische Operation einer reinen - „metaphysikfreien“ im Sinne Cornelius' - Immanenzphilosophie im Sinne des Versuches einer Theorie der Existentialurteile, an die der junge Adorno explizit anknüpfen wird, um Kritik an Husserl zu üben. So schreibt er in seiner Promotionsschrift Zur Transzendenz des Dinglichen und Noematischen in Husserls Phänomenologie (1924): „Vom Standpunkt einer reinen Immanenzphilosophie aus soll in dieser Arbeit Husserls Theorie des Dinges an sich, so wie sie in den 'Ideen zu einer reinen Phänomenologie und phänomenologischen Philosophie' entwickelt ist, geprüft werden" (Adorno, 2003, S. 11). Diesen hier verwendeten Begriff einer „reinen Immanenzphilosophie“ hat Adorno dann sicherlich dem Denken Cornelius entnommen: dabei handelt es sich für Cornelius letztendlich um den methodischen und systematischen Ausschluss jeglicher Inhalte oder Begrifflichkeiten (wie beispielsweise, wie wir sahen, des traditionellen Kausalitäts- und Dingbegriffs), die nicht auf den Bewusstseinsverlauf mit Klarheit und Evidenz zurückgeführt werden können und deshalb als „Bewusstseinstranszendentes“ für Cornelius zu bezeichnen sind.

Zwar sind die unter Cornelius verfassten Arbeiten Adornos grundsätzlich als Schulphilosophie rezipiert, derzufolge Adorno in ihnen bloss die philosophische Position seines Lehrers und Betreuers unkritisch übernommen habe. Dabei verträte er dieselbe Variante vom radikalisierten transzendentalen Idealismus Cornelius', was in klarem Gegensatz zu dem eigentlichen - materialistischen - Philosophieren Adornos steht. Als Schulphilosophie hat zuerst Rolf Tiedemann, der Herausgeber von Adornos Gesammelten Schriften, diese Phase seiner Produktion charakterisiert; die vollzogene Wende zum Materialismus, der eigentliche Anbeginn der Adornoschen Philosophie, wäre dann ihm zufolge bei den sogenannten Vorträgen und Thesen der frühen Dreißiger Jahre zu lokalisieren. In seinen eigenen Worten: 
'Der Begriff des Unbewußten' und die frühe Husserlarbeit sind Schulphilosophie: Arbeiten eines Schülers von Hans Cornelius, von dem heute kaum mehr bekannt ist, als daß Lenin ihn einen Wachtmeister auf dem Professorenkatheder und Flohknacker schimpfte. Sowohl Adornos Dissertation von 1924 - das Rigorosum fand am 28. 7. 1924 vor der Philosophischen Fakultät der Universität Frankfurt a.M. statt - wie der 'Begriff des Unbewußten', den Adorno 1927 derselben Fakultät als Habilitationsschrift einreichte, aber noch vor der Einleitung des Habilitationsverfahrens zurückzog, stellen sich vorbehaltlos auf den Standpunkt der Cornelius'schen Version des transzendentalen Idealismus. (...)

Die im zweiten Teil des Bandes abgedruckten Vorträge und Thesen belegen den vollzogenen Übergang der Adornoschen Philosophie vom transzendentalen Idealismus zum Materialismus; in Wahrheit den Beginn der Adornoschen Philosophie. Er hängt, wenn man denn Namen nennen will, mit der Ablösung von Cornelius und dem Anschluß an Walter Benjamin auf das engste zusammen (Adorno, 2003, S. 382-3). ${ }^{12}$

Doch auch wenn wir diese herrschende Meinung nicht revidieren müssen, ist es nötig zu erwähnen, dass Adorno interessanterweise diesen für sein Denken sehr bedeutenden Begriff lebenslänglich verwendet - auch wenn der Begriff bei dem $12 \quad$ Carlo Pettazzi erzielt letztinstanzlich ähnliche Ergebnisse; diese (Vor-)Periode des Denkens Adornos nennt er übrigens die transzendentale Phase: „Possiamo infatti anticipare i risultati a cui arriveremo schematicamente cosí: la frattura, sia per forma come per contenuto, tra Der Begriff e Kierkegaard è nettissima ed i punto di contatto praticamente assenti; un confronto tra le due tesi di libera docenza e le opere filosofiche successive mostra che quanto il Der Begriff se ne discosta completamente, tanto il Kierkegaard ne anticipa inequivocabilmente motivi essenziali. In questo quadro, quella che noi abbiamo indicata come fase trascendentale, assume il carattere di una parentesi senza conseguenze, di un intermezzo inspiegabile, all'interno dello sviluppo del pensiero di Adorno. Non è infatti facile trovare da indicare elementi della fase trascendentale, della lezione di Cornelius, che influenzino il pensiero dell'Adorno maturo, o che permangano in qualche modo in esso. Se si resto in campo filosofico, non pare che si possa andare al di là di indicazioni estremamente generali, come l'opposizione ad ogni intuizionismo, l'atteggiamento antimetafisico et la diffidenza verso ogni dogmatismo; se si estende l'attenzione all'atteggiamento complessivo verso a cultura si può ricordare anche l'interdisciplinarietà di interessi, la quale però è presente in Adorno già fin dall'adolescenza. Che senso dare dunque all'adesione adorniana alla sistematica trascendentale, si di essa pare resti nelle opere successive poco o nulla? Una prima risposta può essere fornita da quelle motivazioni di carattere pratico che avevamo ipotizzato anche per Horkheimer (...)". Pettazzi, 1977, S. 48-9. 
späten Adorno eine kritische Wende erfährt, da er gegen jede Immanenzphilosophie richtet.

In diesem Sinne: Cornelius' Gesamtpersönlichkeit, das breite Spektrum seiner Interessen und Tätigkeiten, seine sehr progressiven politischen Positionen, seine antidogmatische und aufklärerische Philosophie in Auseinandersetzung und Kritik an den wichtigsten Strömungen der Zeit und - last but not least seine akademische Befürwortung für die Einrichtung eines Instituts für Sozialforschung im Rahmen der Frankfurter Universität machten ihn zu einer außerordentlichen akademischen Figur von höher Wichtigkeit für seine Schüler. In dieser Hinsicht kann Cornelius ohne jede Übertreibung als einen akademischen Vater der Frankfurter Kritischen Theorie bezeichnet werden.

\section{Schluss}

Schließlich, um einen zugleich zusammenfassenden und panoramischen Einblick in das Denken Hans Cornelius' zu geben, sei ein etwas langes, aber höchst instruktives Zitat von Max Horkheimer angeführt, das Teil eines 1923 - das Jahr, da Horkheimer bereits Cornelius' Assistent war und Adorno sich wohl bei der Vorbereitung seiner Dissertation befand - verfassten Textes ist, der sich dem Münchener Philosoph widmet. Diese sehr lehrreiche Textstelle platziert in wenigen Zeilen das Denken Cornelius innerhalb der neuzeitlichen Philosophiegeschichte und hat darüber hinaus den Vorzug, seine Philosophie so darzustellen, wie der junge kritische Theoretiker sie empfang. So schreibt der junge Horkheimer:

Die Humesche Skepsis wurde von Kant überwunden. Cornelius hat die Kantische Lösung aufgenommen und konsequent durchgeführt. Diese Lösung liegt wesentlich im Hinweis auf gewisse fundamentale Beziehungen zwischen den Erlebnissen, die man bis dahin zu studieren unterlassen hatte. Unmittelbar gegeben ist uns nämlich nirgends eine bloße Summe von Einzelerlebnissen, die quasi als selbständige Erwasse, mechanisch aneinandergereiht, einander folgen würden. Dann freilich wären die Regelmäßigkeiten ihres Ablaufes (die Assoziationsgesetze, wie Hume sie aufgestellt hatte) das einzig Gesetzmäßige, was ihre Betrachtung uns enthüllen könnte. Vielmehr zeigen sich die Erlebnisse verbunden zur Einheit des persönlichen Bewußtseins, als dessen Teilinhalte sie sich geben und in Beziehung 
auf das sie immer nur Teilerlebnisse sind. In ihrer Vereinzelung betrachtet sind sie Abstraktionen, ja sogar falsche Abstraktionen. Die Hauptaufgabe der Kantischen Transzendentalphilosophie, so wie sie von Cornelius verstanden und gelehrt wird, ist es, die Beziehungen zu studieren, die für die Einheit unseres Bewußtseins konstitutiv sind, und die Faktoren herauszustellen, die notwendige Bedingungen für den Zusammenhang unseres Bewußtseins bilden. Die allgemeinsten Gesetze, die aus einer solchen Betrachtung sich ergeben, müssen dann solche sein, ohne die ein Bewußtseinszusammenhang nicht gedacht werden kann, und die daher grundlegend sind für alle künftige Erfahrung. Das Studium dieser Gesetzäßigkeiten und auf Grund dieses Studiums die endgültige Klärung der schon erwähnten Grundbegriffe: des beharrlichen Dings, der Kausalität, der Persönlichkeit durch eingehende Bedeutungsanalysen ist Ziel und Inhalt der Corneliusschen Philosophie (...) (Horkheimer, 1979, S. 151-2).

\section{Literaturverzeichnis}

ADORNO, T. Zur Transzendenz des Dinglichen und Noematischen in Husserls Phänomenologie, in: Gesammelte Schriften 1, Frankfurt am Main: Suhrkamp 2003.

CORNELIUS, C. Versuch einer Theorie der Existentialurteile. München, 1894.

CORNELIUS, C. Psychologie als Erfahrungswissenschaft. Leipzig: Teubner 1897.

CORNELIUS, C. Leben und Lehre, Felix Meiner, Leipzig 1921.

CORNELIUS, C. Zur Kritik der wissenschaftlichen Grundbegriffe, in: Hans Reichenbach \& Rudolf Carnap (Hrgs.), Erkenntnis, Springer, 1931.

HORKHEIMER, M. Hans Cornelius. Zu seinem 60. Geburtstag, in: Gesammelte Schriften 2, Fischer, 1979.

HORKHEIMER, M. Kritische Theorie gestern und heute, in: Gesammelte Schriften 8, Frankfurt am Main: Fischer, 1979.

HUSSERL, E. Logische Untersuchungen. Zweiter Band, Erster Teil: Untersuchungen zur Phänomenologie und Theorie der Erkenntnis. Den Haag, 1921.

HUSSERL, E. Aufsätze und Rezensionen (1890-1910). Hua XXII, Martinus Nijhoff, Den Haag, 1979.

JAY, M. The dialectical imagination: A History of the Frankfurt School and the Institute of Social Research, 1923-1950, University of California Press, 1996.

PETTAZZI, C. Studien zu Leben und Werk Adornos bis 1938. in: Text und Kritik 56. München, 1977. 
PETTAZZI, C. Theodor Wiesengrund-Adorno: Linee di origine e sviluppo del pensiero. Roma: La Nuova Italia, 1979.

ROLOLINGER, R. Husserl and Cornelius, in: Husserl Studies 8, 1991. SCHMIDT, R. (Hrg.). Die Philosophie der Gegenwart in Selbstdarstellungen 2, Leipzig: Meiner, 1921.

SMID, R. N. „Münchener Phänomenologie“ - Zur Frühgeschichte des Begriffs, in: Herbert Spiegelberg und Eberhard Avé-Lallemant (Hrgs.). Pfänder-Studien, Martinus Nijhoff, 1982. 